\title{
Charge Transfer Polycondensation of 1,4-Bis(methoxycarbonylethyl)- piperazine with Hexamethylenediamine in the Presence of Nitrated Polystyrene
}

\author{
Naoya OGata, Kohei Sanui, Masakazu YoshiKawa, \\ Hiroyuki BABA, and Kazuhide Goto \\ Department of Chemistry, Faculty of Science and Technology, \\ Sophia University, 7-1 Kioi-cho, Chiyoda-ku, \\ Tokyo 102, Japan
}

(Received September 13, 1984)

\begin{abstract}
Polycondensation of 1,4-bis(methoxycarbonylethyl)piperazine (MCEP) with hexamethylenediamine (HMD) was carried out in the presence of an electron-accepting matrix such as a nitrated polystyrene. A charge transfer complex was found to be formed between HMD and the matrix polymer and the rate of the polycondensation was enhanced by the adsorption of HMD in the matrix polymer of nitrated polystyrene.
\end{abstract}

KEY WORDS Polycondensation / Charge Transfer / Matrix Polycondensation / Nitrated Polystyrene /

It has already been reported ${ }^{1}$ that the rate of the polycondensation of dimethyl tartarate (DMT) with hexamethylenediamine (HMD) is enhanced by the presence of polymer matrices such as poly(1-vinylpyrrolidone), polysaccharide, or poly(vinyl alcohol). This rate enhancement is due to the hydrogen-bonding interaction between DMT or the polyamide and matrix polymer so that monomers are adsorbed along the chains of matrix polymers, thus increasing the local concentration of monomers at the reaction stage of the polycondensation. The presence of poly(4vinylpyridine) did not enhance the rate of the polycondensation of diethyl mucate with HMD, but the molecular weight of the resulting polyamide increased. ${ }^{2}$

The hydrogen-bonding interaction is a potential interaction for matrix polycondensation. A charge transfer interaction may be expected to be the same. The polycondensation of diethyl chelidonate with diamines in the presence of polyvinylcarbazole was carried out as a matrix polycondensation by the charge transfer interaction. ${ }^{3}$

This article described the results of the polycondensation of 1,4-bis(methoxycarbonylethyl)piperazine (MCEP) with HMD in the presence of electron-accepting nitrated polystyrene (NPS), which may possibly form a charge transfer complex with electrondonating monomers.

It was previously found ${ }^{4}$ that 1,4-bis(methoxycarbonylmethyl)piperazine reacts
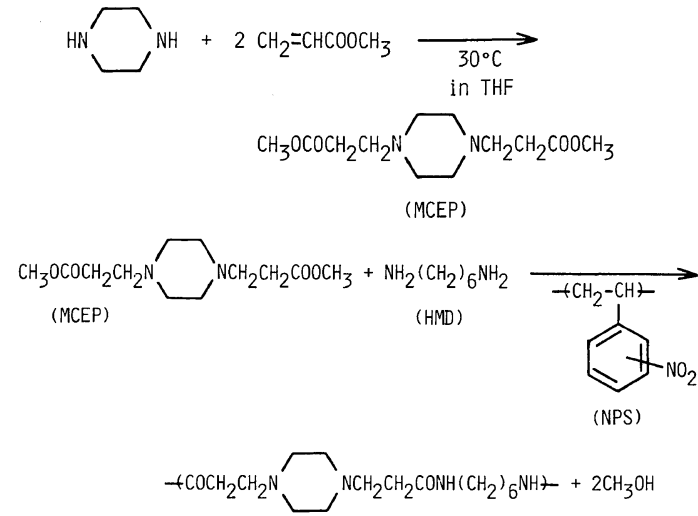
with HMD under mild conditions to form the corresponding polyamide. Since MCEP contains binary amine groups which are electrondonating, either MCEP or HMD may form a charge transfer with NPS. Therefore, the complex formation between MCEP or HMD was investigated to clarify the effects of NPS on the polycondensation of MCEP and HMD.

\section{EXPERIMENTAL}

\section{Materials}

Methyl acrylate, piperazine, styrene, 2,2'azobisisobutyronitrile (AIBN), tetrahydrofuran (THF) hexamethylenediamine (HMD), benzene, $N, N$-dimethyformamide (DMF), and $N, N$-dimethylacetamide (DMAc) were purified in the usual manner. Ligroin, diethyl ether, methanol, and tetrachloromethane were used without purification. Polystyrene, PS-2, having a reduced viscosity of 0.86 , was purchased from Wako Pure Chemical Industries, Ltd. and reprecipitated in benzene/methanol.

\section{Synthesis of 1,4-Bis(methoxycarbonylethyl)-} piperazine (MCEP)

MCEP was synthesized from piperazine and methyl acrylate in THF below $30^{\circ} \mathrm{C} .^{5}$ Elemental analysis of MCEP showed close agreement with the calculated values as follows: C, $56.1 \%$; H, $8.6 \%$; N, $11.1 \%$. Calcd for $\mathrm{C}_{12} \mathrm{H}_{22} \mathrm{~N}_{2} \mathrm{O}_{4}$ : C, $55.78 \% ; \quad \mathrm{H}, \quad 8.60 \% ; \quad \mathrm{N}$, $10.85 \%$.

\section{Synthesis of Polystyrene (PS-I)}

PS-1 was synthesized as follows: $120 \mathrm{~g}$ $(1.152 \mathrm{~mol})$ of styrene, $0.0946 \mathrm{~g} \quad(5.76 \times$ $10^{-3} \mathrm{~mol}$ ) of AIBN, and $150 \mathrm{~cm}^{3}$ of benzene were placed in an ampule and sealed under a nitrogen atmosphere. The polymerization was carried out at $50^{\circ} \mathrm{C}$ over a period of $48 \mathrm{~h}$. Reprecipitation with methanol gave $63.8 \mathrm{~g}$ $(53.2 \%)$ of PS- 1 .

\section{Nitration of Polystyrene \\ Nitration of polystyrene was carried out}

according to the method of Blanchette and Cotman. ${ }^{6}$

The amount of incorporated nitro groups was adjusted by the following reaction conditions: NPS-1 was obtained by the reaction of fuming nitric acid and PS-1 in tetrachloromethane below $40^{\circ} \mathrm{C}$. NPS- 2 and NPS- 4 were obtained by nitration of PS-1 and PS- 2 with fuming nitric acid below $0^{\circ} \mathrm{C}$, respectively. NPS-3 was obtained by the nitration of PS-1 with fuming nitric acid at $50^{\circ} \mathrm{C}$. The results are summarized in Table I.

\section{Measurement of Absorption Spectra}

The absorption spectra were measured by a Shimadzu UV-240 UV-Visible Recording Spectrophotometer. Polymer concentration was defined as the molar concentration of the substituted nitro moiety. Difference absorption spectra were measured after making exactly equal the concentrations of the optical path of the reference and sample. Interactions between NPS-2, -3 , and -4 and HMD were observed by mixing $2.00 \times 10^{-3} \mathrm{~mol} \mathrm{dm}^{-3}$ DMF solution of NPS with HMD. $2.00 \times 10^{-2}$ mol dm ${ }^{-3}$ DMF solution was used in case of NPS-1 and HMD.

\section{Polycondensation}

The polycondensation reaction of MCEP with HMD was carried out in both the presence and absence of NPS in DMF by a conventional polycondensation method at

Table I. Results of matrix polymer synthesis

\begin{tabular}{lccc}
\hline & Yield & & \\
\cline { 2 - 3 } Sample & $\%$ & $\eta_{\text {sp }} / c^{\mathrm{a}}$ & $\mathrm{NO}_{2} / \mathrm{C}_{6} \mathrm{H}_{5}{ }^{\mathrm{b}}$ \\
& $\%$ & & \\
\hline PS-1 & 53.2 & 0.29 & - \\
PS-2 & 96.6 & 0.86 & - \\
NPS-1 & 50.0 & 0.23 & 0.48 \\
NPS-2 & 87.8 & 0.21 & 1.09 \\
NPS-3 & 73.9 & 0.12 & 1.53 \\
NPS-4 & 73.9 & 0.67 & 1.06 \\
\hline
\end{tabular}

a $0.1 \mathrm{~g} / 10 \mathrm{~cm}^{3}$ PS in benzene and NPS in DMAc at $30^{\circ} \mathrm{C}$.

${ }^{b}$ Determined by elemental analysis. 
$60^{\circ} \mathrm{C}$. The added amount of NPS was adjusted according to the concentration of repeating units based on the amount of substituted nitro moiety. The rates of polycondensation were followed by measuring the amount of liberated methanol on a Shimadzu GC-7AG Chromatograph equipped with a 1 m-long column with Tenax GC. The polycondensation was initiated by mixing a DMF solution of MCEP with that of HMD and NPS. This system was allowed to stand overnight in order to reach an equilibrium and form a charge transfer complex between HMD and NPS.

Separation of polyamide from the matrix, NPS, was carried out by extracting the obtained polyamide with methanol in a Soxhlet extraction apparatus.

\section{RESULTS AND DISCUSSION}

\section{Interaction between MCEP and Matrix} Polymer, NPS

Figure 1 indicates difference absorption spectra of various solutions containing NPS-2 as a matrix polymer. When NPS- 2 and HMD were mixed, a new absorption band appeared indicating that a charge transfer complex had been formed between NPS-2 containing electron-accepting $\mathrm{NO}_{2}$ moiety, and HMD having an electron-donating character. No new absorption band around $439 \mathrm{~nm}$ was observed on mixing NPS-2 and MCEP. Therefore, a charge transfer complex of NPS may be formed not with MCEP, but with HMD.

The Benesi-Hildebrandt $\operatorname{plot}^{7}$ was obtained from the difference absorbance, $\triangle O D$, as shown in Figure 2 based on the BenesiHildebrandt equation as follows,

$$
\begin{aligned}
\frac{\left[\mathrm{NO}_{2}\right]}{\Delta \mathrm{OD}}= & \frac{1}{[\mathrm{HMD}]} \frac{1}{K \varepsilon}+\frac{1}{\varepsilon} \\
& \varepsilon=\varepsilon_{\mathrm{B}}-\varepsilon_{\mathrm{F}}
\end{aligned}
$$

where $\Delta \mathrm{OD}$ is the difference absorbance. $K$, the binding constant. $\left[\mathrm{NO}_{2}\right]$ and $[\mathrm{HMD}]$, the con-

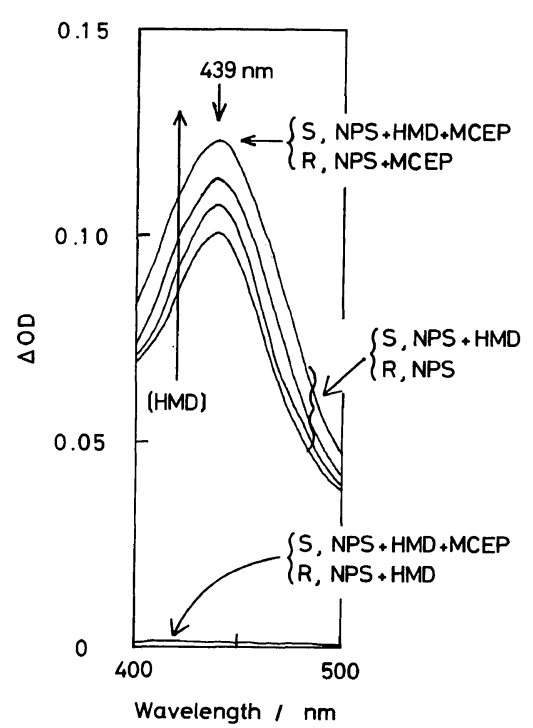

Figure 1. Difference absorption spectra of various solution systems. $\left[\mathrm{NO}_{2}\right], 2.00 \times 10^{-3}$ unit mol dm $\mathrm{dm}^{-3}$; [HMD], $2.06 \times 10^{-2}, 2.57 \times 10^{-2}, 3.30 \times 10^{-2}$, or $4.99 \times$ $10^{-2} \mathrm{~mol} \mathrm{dm}^{-3}$.

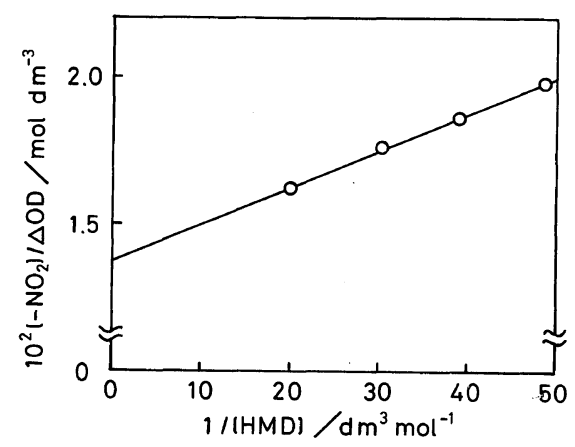

Figure 2. Benesi-Hildebrandt relationship for the binding of HMD to NPS. [ $\mathrm{NO}_{2}$ ], $2.00 \times 10^{-3}$ unit mol $\mathrm{dm}^{-3} ; \Delta \mathrm{OD}$, the difference optical density at $439 \mathrm{~nm}$; $K=110 \mathrm{dm}^{3} \mathrm{~mol}^{-1} ; \varepsilon=72.8$.

centrations of $\mathrm{NO}_{2}$ and $\mathrm{HMD}$, respectively and $\varepsilon_{\mathrm{B}}$ and $\varepsilon_{\mathrm{F}}$ are the extinction coefficients of the nitro moiety bound by HMD and free nitro moiety, respectively. From the intercept and slope in Figure 2, $K$ and $\varepsilon$ values were obtained. A rearranged form of the Langmuir isotherm derived by Klotz et al. ${ }^{8}$ can also be applied to the present system. 


$$
\begin{gathered}
\frac{[\mathrm{HMD}]}{\left[\mathrm{NO}_{2}\right]_{\mathrm{B}}}=\frac{1}{\left[\mathrm{NO}_{2}\right]_{\mathrm{F}}} \frac{1}{K^{\prime} n}+\frac{1}{n} \\
{\left[\mathrm{NO}_{2}\right]_{\mathrm{B}}=\Delta \mathrm{OD} / \varepsilon}
\end{gathered}
$$

In eq $2,\left[\mathrm{NO}_{2}\right]_{\mathrm{B}}$ and $\left[\mathrm{NO}_{2}\right]_{\mathrm{F}}$ denote the concentration of the nitro moiety bound by HMD and that of free nitro moiety, respectively $\left(\left[\mathrm{NO}_{2}\right]=\left[\mathrm{NO}_{2}\right]_{\mathrm{B}}+\left[\mathrm{NO}_{2}\right]_{\mathrm{F}}\right) . K^{\prime}$ is the intrinsic binding constant; the constant $n$ denotes the minimum number of $\mathrm{NO}_{2}$ required to bind the HMD molecule. Figure 3 shows the Klotz plot; $K^{\prime} n$ and $n$ were also evaluated from the intercept and slope. Obtained values, $\varepsilon$ and $K$ from the Benesi-Hildebrandt relation, and $n$, $K^{\prime} n, K^{\prime}$ from the Klotz relation for all NPSHMD systems are summarized in Table II which indicates the $K$ values to be in close agreement with $K^{\prime} n$. The constant $n$ in each system was determined as 2 . This suggests that the nitro moiety in NPS interacts with the amine group in HMD to form a one-to-one complex.

On the other hand, the interaction between NPS and MCEP was scarcely observed by spectroscopic measurement. It was concluded that the interaction between MCEP and NPS was very weak compared to that between HMD and NPS.

These results suggest that an enhancement of the polycondensation reaction of MCEP with HMD takes place in the presence of NPS owing to the formation of the charge transfer

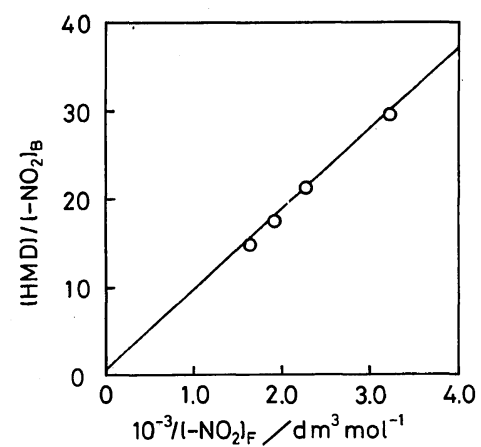

Figure 3. Klotz relationship for the binding of HMD to NPS. $K^{\prime} n=110 \mathrm{dm}^{3} \mathrm{~mol}^{-1} ; n=2 ; K^{\prime}=55 \mathrm{dm}^{3} \mathrm{~mol}^{-1}$. interaction during polycondensation.

\section{Polycondensation}

Figure 4 shows the time-conversion curves for the polycondensation of MCEP with HMD in DMF at a monomer concentration of $0.02 \mathrm{~mol} \mathrm{dm}^{-3}$ in the presence or absence of NPS at $60^{\circ} \mathrm{C}$. As shown in Figure 4, the polycondensation of MCEP with HMD took place very slowly with a conversion of only $10 \%$ after 5 days, but was enhanced by the presence of NPS. Rate enhancement caused by the NPS matrix increased in proportion to the mol fraction of substituted nitro moiety in NPS. Conversion of the polycondensation reaction reached $100 \%$ after 5 days in the presence of NPS-3. The apparent rate constants of the present matrix polycondensation were calculated as those of second-ordered reactions and are summarized in Table III.

Table II. Binding constants between HMD

\begin{tabular}{|c|c|c|c|c|c|}
\hline \multirow{2}{*}{ Matrix } & \multirow{2}{*}{$\varepsilon$} & $K$ & \multirow{2}{*}{$n$} & $K^{\prime} n$ & $K^{\prime}$ \\
\hline & & $\mathrm{dm}^{3} \mathrm{~mol}^{-1}$ & & $\mathrm{dm}^{3} \mathrm{~mol}^{-1}$ & $\mathrm{dm}^{3} \mathrm{~mol}^{-1}$ \\
\hline NPS-1 & 7.1 & 77.4 & 2 & 80.5 & 40.3 \\
\hline NPS-2 & 72.8 & 110 & 2 & 110 & 55 \\
\hline NPS-3 & 402 & 489 & 2 & 469 & 235 \\
\hline NPS-4 & 93.6 & 63.4 & 2 & 63.6 & 31.8 \\
\hline
\end{tabular}
and the matrices

Benesi-Hildebrand: $\left[\mathrm{NO}_{2}\right] / \Delta \mathrm{OD}=1 /[\mathrm{HMD}] K \varepsilon+1 / \varepsilon$. Klotz: $\left[\mathrm{NO}_{2}\right]_{\mathrm{B}} /[\mathrm{HMD}]=1 /\left[\mathrm{NO}_{2}\right]_{\mathrm{F}} K^{\prime} n+1 / n$.

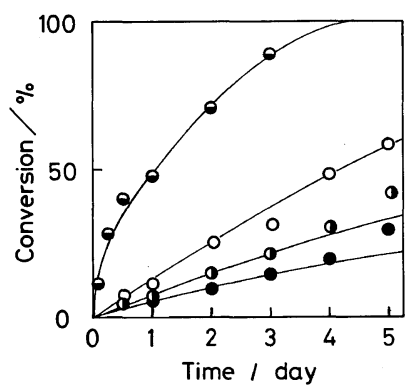

Figure 4. Effects of various matrices on polycondensation of MCEP with HMD in DMF at $60^{\circ} \mathrm{C}$. [Monomer], $2.0 \times 10^{-2} \mathrm{~mol} \mathrm{dm}{ }^{-3} ;\left[\mathrm{NO}_{2}\right] /\left[\mathrm{NH}_{2}\right], 1 ; \mathbf{O}$, None; 1 , NPS-1; $\bigcirc$, NPS-2; $\ominus$, NPS-3. 
Table III. Rate constants $(k)$ of the matrix polycondensation of MCEP with HMD in $\mathrm{DMF}$ at $60^{\circ} \mathrm{C}^{\mathrm{a}}$

\begin{tabular}{cccc}
\hline \multirow{2}{*}{ Matrix } & \multicolumn{1}{c}{$K$} & \multicolumn{1}{c}{$k$} \\
\cline { 2 - 2 } & $\mathrm{dm}^{3} \mathrm{~mol}^{-1}$ & & $\mathrm{dm}^{3} \mathrm{~mol}^{-1} \mathrm{~h}^{-1}$ \\
\hline None & - & & $1.17 \times 10^{-1}$ \\
NPS-1 & 77.4 & & $2.28 \times 10^{-1}$ \\
NPS-2 & 110 & & $2.78 \times 10^{-1}$ \\
NPS-3 & 489 & 3.40 \\
\hline
\end{tabular}

a Monomer concn, $0.02 \mathrm{~mol} \mathrm{dm}^{-3}$.

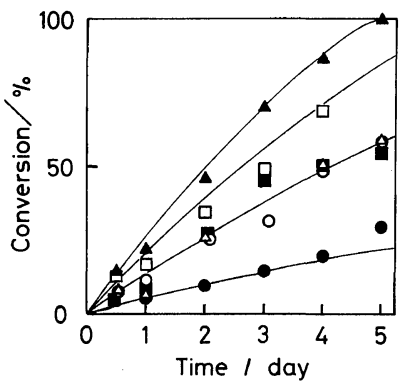

Figure 5. Matrix polycondensation of MCEP with HMD in DMF at $60^{\circ} \mathrm{C}$. Matrix, NPS-2; [Monomer], $2.0 \times 10^{-2} \mathrm{~mol} \mathrm{dm}^{-3}$. [ $\left.\mathrm{NO}_{2}\right] /\left[\mathrm{NH}_{2}\right]:(\bigcirc) 0.00 ;(\triangle) 0.31$;

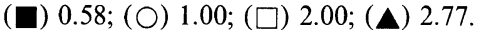

Table IV. Rate constants $(k)$ of the matrix polycondensation of MCEP with HMD in $\mathrm{DMF}^{\mathrm{a}}$ at $60^{\circ} \mathrm{C}$

\begin{tabular}{|c|c|c|}
\hline \multirow{2}{*}{$\mathrm{NO}_{2} / \mathrm{NH}_{2}$} & Temp & $k$ \\
\hline & ${ }^{\circ} \mathrm{C}$ & $\mathrm{dm}^{3} \mathrm{~mol}^{-1} \mathrm{~h}^{-1}$ \\
\hline 0.00 & 60 & $1.17 \times 10^{-1}$ \\
\hline 0.31 & 60 & $3.33 \times 10^{-1}$ \\
\hline 0.58 & 60 & $2.52 \times 10^{-1}$ \\
\hline 1.00 & 60 & $2.78 \times 10^{-1}$ \\
\hline 2.00 & 60 & $5.93 \times 10^{-1}$ \\
\hline 2.77 & 60 & 1.37 \\
\hline
\end{tabular}

a Monomer concn, $0.02 \mathrm{~mol} \mathrm{dm}^{-3}$; matrix, NPS-2.

Figure 5 shows the effect of the concentration of NPS-2 on the polycondensation of MCEP with HMD. The apparent secondordered rate constants were calculated as summarized in Table IV. A prominent rate enhancement of polycondensation was observed in the presence of NPS matrix when the ratio of $\left[\mathrm{NH}_{2}\right] /\left[\mathrm{NO}_{2}\right]$ was over 1 . This enhancement effect may be attributed to the increase in the amount of adsorbed monomer, HMD, along the NPS matrix owing to the charge transfer interaction, possibly resulting in an increase in the local concentration of HMD at the reaction phase to enhance the polycondensation reaction.

Polymers were isolated from the matrix polymer and the reduced viscosities of the obtained polyamides were measured in DMAc. $\eta_{\mathrm{sp}} / c$ values were less than 0.1 (Reduced viscosity was measured at $c=0.1 \mathrm{~g} /$ $10 \mathrm{~cm}^{3}$ in DMAc at $30^{\circ} \mathrm{C}$.). The low solution viscosities of these polyamides may be caused by the very low concentration of $0.02 \mathrm{~mol}$ $\mathrm{dm}^{-3}$ of the monomer which did not influence the molecular weight increase of resulting polyamide, although the polycondensation rates were enhanced in the presence of NPS. It is expected that an increase in the molecular weight of the resulting polyamide may become evident with increasing concentration of monomer and matrix polymer above $0.1 \mathrm{~mol}$ $\mathrm{dm}^{-3}$. However, the solubility of these compounds in DMF was not high and experiments at higher concentrations were not carried out.

\section{REFERENCES}

1. N. Ogata, K. Sanui, H. Nakamura, and $\mathbf{M}$. Kuwahara, J. Polym. Sci., Polym. Chem. Ed., 18, 939 (1980).

2. N. Ogata, K. Sanui, and H. Nakamura, J. Polym. Sci., Polym. Chem. Ed., 18, 933 (1980).

3. N. Ogata, K. Sanui, and M. Abe, J. Polym. Sci., Polym. Chem. Ed., 19, 1361 (1981).

4. N. Ogata, Y. Hosoda, and G. Suzuki, Polym. J., 6, 412 (1974).

5. M. Sumoto and T. Isozaki, Kogyo Kagaku Zasshi, 68, 1989 (1965).

6. J. A. Blanchette and J. D. Cotman, Jr., J. Am. Chem. Soc., 80, 1117 (1958).

7. H. A. Benesi and J. H. Hildebrandt, J. Am. Chem. Soc., 71, 2703 (1949).

8. I. M. Klotz, F. M. Waiker, and R. B. Pivan, J. Am. Chem. Soc., 68, 1486 (1946). 